\title{
A Spherical Microphone Array System for Traffic Scene Analysis
}

\author{
Zhiyun Li, Ramani Duraiswami, Elena Grassi, Larry S. Davis
}

\begin{abstract}
This paper describes a practical spherical microphone array system for traffic auditory scene capture and analysis. Our system uses 60 microphones positioned on the rigid surface of a sphere. We then propose an optimal design of a robust spherical beamformer with minimum white noise gain (WNG) of $-6 \mathrm{~dB}$. We test this system in a real-world traffic environment. Some preliminary simulation and experimental results are presented to demonstrate its performance. This system may also find applications in broader areas such as 3D audio, virtual environment, etc.
\end{abstract}

\section{INTRODUCTION}

Recently computer vision based traffic surveillance has been attracting many researchers [1][2][3][4]. On the other hand, the sound from a running vehicle also provides rich information about the vehicle. Researchers have used analogous methods from computer vision for vehicle sound signature recognition [5]. To extract spatial information from the auditory scene, however, we need a microphone array, such as the SAS-1 system [6]. Spherical arrays of microphones are recently becoming the subject of some study as they allow omnidirectional sampling of the soundfield [8][9], and seeing potential applications in spatial soundfield analysis. In this paper, we will build a practical spherical microphone array system and use it for real-world traffic auditory scene capture and analysis.

This paper is organized into four parts: first, we briefly overview our system; we then present the theory of spherical beamformer which forms the basis of our system; third, we will design an optimal and robust beamformer for our array under the constraint of minimum $-6 \mathrm{~dB}$ white noise gain; in the fourth part, we will demonstrate the performance of our system by simulations and real-world experiments.

\section{SYSTEM OVERVIEW}

Our system is as shown in Fig. 1. The main part is the spherical microphone array which consists of 60 omnidirectional microphones mounted on the rigid spherical surface of radius $10 \mathrm{~cm}$. The positions of the 60 microphones are decided as the 64 nodes in [10] with four nodes removed because of the cable outlet and the mounting base. In fact, the layout also can be flexible [13]. The sound signals received by the array will flow through a 64 channel preamplifier before acquired by two 32 channel NI-DAQ cards on the computer.

This work was partially supported by NSF Award 0205271 .

Perceptual Interfaces and Reality Laboratory, UMLACS, University of Maryland, College Park, MD 20742. Email: zliecs.umd. edu

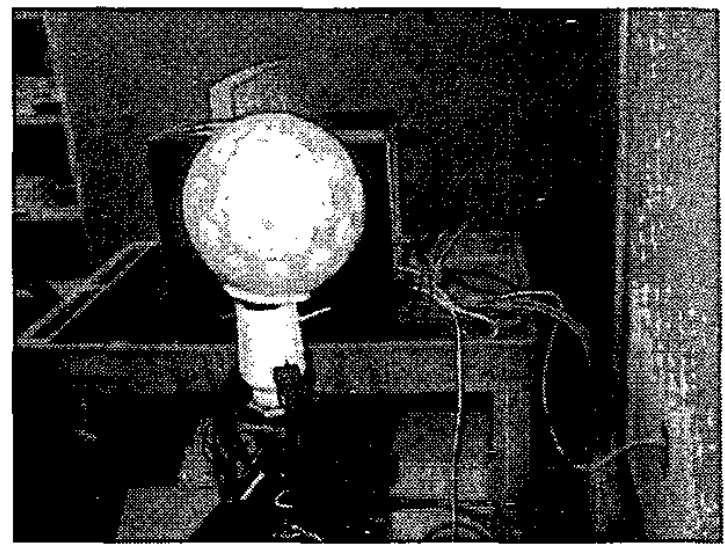

Fig. 1. The snapshot of our spherical microphone array system.

\section{THEORY OF SPHERICAL BEAMFORMER}

The signal processing unit of our system is a spherical beamformer which is a spatial filter of 3D space. The basic principle of spherical beamformer is to make use of the orthonormality of spherical harmonics to decompose the soundfield arriving at a spherical array. Then the orthogonal components of the soundfield are linearly combined to approximate a desired beampattern.

\section{A. Scattering from A Rigid Sphere}

For a unit magnitude plane wave $\mathbf{k}$, incident from direction $\left(\theta_{k}, \varphi_{k}\right)$, the incident field at a point $\left(\theta_{s}, \varphi_{s}, r_{s}\right)$ is

$$
\begin{aligned}
p_{i}= & e^{i \mathbf{k} \cdot \mathbf{r}_{s}} \\
= & 4 \pi \sum_{n=0}^{\infty} i^{n} j_{n}\left(k r_{s}\right) \\
& \times \sum_{m=-n}^{n} Y_{n}^{m}\left(\theta_{k}, \varphi_{k}\right) Y_{n}^{m *}\left(\theta_{s}, \varphi_{s}\right),
\end{aligned}
$$

where $j_{n}$ is the spherical Bessel function of order $n, Y_{n}^{m}$ is the spherical harmonics of order $n$ and degree $m .{ }^{*}$ denotes the conjugation. At the same point, the field scattered by the rigid sphere of radius $a$ is [7]:

$$
\begin{aligned}
p_{s}=- & 4 \pi \sum_{n=0}^{\infty} i^{n} \frac{j_{n}^{\prime}(k a)}{h_{n}^{\prime}(k a)} h_{n}\left(k r_{s}\right) \\
& \times \sum_{m=-n}^{n} Y_{n}^{m}\left(\theta_{k}, \varphi_{k}\right) Y_{n}^{m *}\left(\theta_{s}, \varphi_{s}\right) .
\end{aligned}
$$


The total field on the surface $\left(r_{s}=a\right)$ of the rigid sphere is:

$$
\begin{gathered}
p_{t}=\left.\left(p_{s}+p_{i}\right)\right|_{r_{s}=a} \\
=4 \pi \sum_{n=0}^{\infty} i^{n} b_{n}(k a) \sum_{m=-n}^{n} Y_{n}^{m}\left(\theta_{k}, \varphi_{k}\right) Y_{n}^{m *}\left(\theta_{s}, \varphi_{s}\right), \\
b_{n}(k a)=j_{n}(k a)-\frac{j_{n}^{\prime}(k a)}{h_{n}^{\prime}(k a)} h_{n}(k a),
\end{gathered}
$$

where $h_{n}$ is the spherical Hankel function of the first kind.

\section{B. Soundfield Decomposition and Beamforming}

If we assume that the pressure recorded at each point $\left(\theta_{s}, \varphi_{s}\right)$ on the surface of the sphere $\Omega_{s}$, is weighted by

$$
W_{n^{\prime}}^{m^{\prime}}\left(\theta_{s}, \varphi_{s}, k a\right)=\frac{Y_{n^{\prime}}^{m^{\prime}}\left(\theta_{s}, \varphi_{s}\right)}{4 \pi i^{n^{\prime}} b_{n^{\prime}}(k a)} .
$$

Then making use of orthonormality of spherical harmonics:

$$
\int_{\Omega_{s}} Y_{n}^{m *}\left(\theta_{s}, \varphi_{s}\right) Y_{n^{\prime}}^{m^{\prime}}\left(\theta_{s}, \varphi_{s}\right) d \Omega_{s}=\delta_{n n^{\prime}} \delta_{m m^{\prime}}
$$

the total output from a pressure-sensitive spherical surface is:

$$
P=\int_{\Omega_{s}} p_{t} W_{n^{\prime}}^{m^{\prime}}\left(\theta_{s}, \varphi_{s}, k a\right) d \Omega_{s}=Y_{n^{\prime}}^{m^{\prime}}\left(\theta_{k}, \varphi_{k}\right)
$$

This shows the gain of the plane wave coming from $\left(\theta_{k}, \varphi_{k}\right)$, for a continuous pressure-sensitive spherical microphone is $Y_{n^{\prime}}^{m^{\prime}}\left(\theta_{k}, \varphi_{k}\right)$. Since an arbitrary real function $F(\theta, \varphi)$ can be expanded in terms of complex spherical harmonics, we can implement arbitrary beampatterns. For example, an ideal beampattern directed at a direction $\left(\theta_{0}, \varphi_{0}\right)$ :

$$
F(\theta, \varphi)= \begin{cases}1, & (\theta, \varphi)=\left(\theta_{0}, \varphi_{0}\right) \\ 0, & \text { otherwise }\end{cases}
$$

can be expanded into:

$$
F(\theta, \varphi)=2 \pi \sum_{n=0}^{\infty} \sum_{m=-n}^{n} Y_{n}^{m *}\left(\theta_{0}, \varphi_{0}\right) Y_{n}^{m}(\theta, \varphi) .
$$

Apparently, this system can be steered into any 3D directions digitally with the same beampattern. This provides the advantage to process sound from every directions unbiasedly.

\section{Optimal Design of Robust Spherical BEAMFORMER}

To have an ideal beampattern for our array with unbalanced microphone layout, we need:

$$
\mathbf{A} \times \mathbf{W}=\mathbf{B}
$$

where $\mathbf{A}$ is the coefficients of the spherical harmonics expansion of the sound field in (5):

$$
\mathbf{A}=\left[\begin{array}{llll}
\mathbf{A}_{1} & \mathbf{A}_{2} & \cdots & \mathbf{A}_{S}
\end{array}\right],
$$

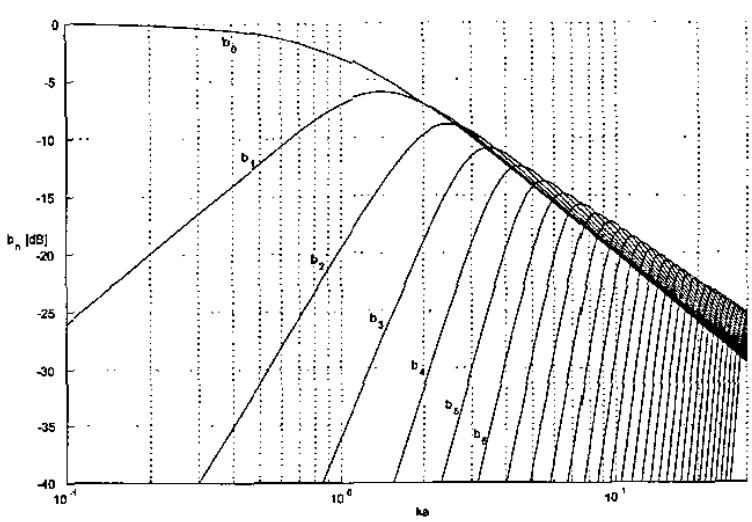

Fig. 2. $b_{n}(k a)$ for orders from 0 to 30 .

$$
\begin{gathered}
\mathbf{A}_{s}=\left[\begin{array}{c}
i^{0} b_{0}(k a) Y_{0}^{0 *}\left(\theta_{s}, \varphi_{s}\right) \\
i^{1} b_{1}(k a) Y_{1}^{-1 *}\left(\theta_{s}, \varphi_{s}\right) \\
\cdots \\
i^{N} b_{N}(k a) Y_{N}^{N *}\left(\theta_{s}, \varphi_{s}\right) \\
\ldots \\
i^{\infty} b_{\infty}(k a) Y_{\infty}^{\infty *}\left(\theta_{s}, \varphi_{s}\right)
\end{array}\right], \\
(s=1, \ldots, S ; \quad \text { in our system } S=60) .
\end{gathered}
$$

and $\mathbf{W}$ is the complex weight to be assigned to each microphones.

$$
\mathbf{W}=\left[\begin{array}{c}
W\left(\theta_{1}, \varphi_{1}\right) \\
W\left(\theta_{2}, \varphi_{2}\right) \\
\cdots \\
W\left(\theta_{S}, \varphi_{S}\right)
\end{array}\right],
$$

$\mathbf{B}$ is the coefficient of the ideal beampattern steering at $\left(\theta_{0}, \varphi_{0}\right)$ in $(11)$ :

$$
\mathbf{B}=\left[\begin{array}{c}
Y_{0}^{0 *}\left(\theta_{0}, \varphi_{0}\right) \\
Y_{1}^{-1 *}\left(\theta_{0}, \varphi_{0}\right) \\
\ldots \\
Y_{N}^{N *}\left(\theta_{0}, \varphi_{0}\right) \\
\ldots \\
Y_{\infty}^{\infty *}\left(\theta_{0}, \varphi_{0}\right)
\end{array}\right] .
$$

In practice, we can only achieve the beampattern to limited orders with 60 microphones. In that case, (12) becomes an over- or under-determined linear system with respect to $\mathbf{W}$.

A robust beamformer requires the minimum WNG is $-6 \mathrm{~dB}$ [12]. So to design an optimal and robust spherical beamformer with limited microphones, we need to optimize the following:

$$
\text { minimize }|\mathbf{A} \times \mathbf{W}-\mathbf{B}|^{2}
$$

subject to:

$$
W N G=10 \log _{10}\left(\frac{\left|\mathbf{W}^{H} \mathbf{d}\right|^{2}}{\mathbf{W}^{H} \mathbf{W}}\right) \geq-6 \mathrm{~dB},
$$




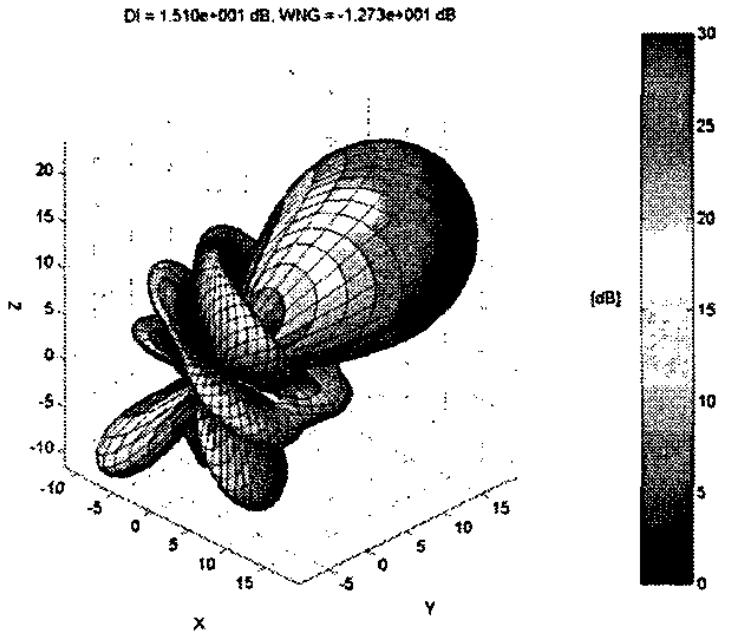

Fig. 3. The unoptimized beampattern of order five at $2 \mathrm{kHz}$. ( $D I=$ $15.1 \mathrm{~dB}, W N G=-12.73 \mathrm{~dB})$.

where $\mathbf{d}$ is the vector of complex pressure on the microphone positions produced by the unit magnitude plane wave from $\left(\theta_{0}, \varphi_{0}\right)$. However, the WNG is also caused by the fast decay of $b_{n}(k a)$ with increasing $n$ as shown in Fig. 2. Consider the following equation:

$$
\begin{aligned}
& {\left[\begin{array}{c}
i^{N} b_{N}(k a) Y_{N}^{N *}\left(\theta_{1}, \varphi_{1}\right) \\
i^{N} b_{N}(k a) Y_{N}^{N *}\left(\theta_{2}, \varphi_{2}\right) \\
\ldots \\
i^{N} b_{N}(k a) Y_{N}^{N *}\left(\theta_{S}, \varphi_{S}\right)
\end{array}\right]^{T} \times\left[\begin{array}{c}
W\left(\theta_{1}, \varphi_{1}\right) \\
W\left(\theta_{2}, \varphi_{2}\right) \\
\ldots \\
W\left(\theta_{S}, \varphi_{S}\right)
\end{array}\right]} \\
& =Y_{N}^{N *}\left(\theta_{0}, \varphi_{0}\right)
\end{aligned}
$$

Using Cauchy's Inequality, we have:

$$
\begin{aligned}
& \left|\sum_{s=1}^{S} i^{N} b_{N}(k a) Y_{N}^{N *}\left(\theta_{s}, \varphi_{s}\right) W\left(\theta_{s}, \varphi_{s}\right)\right|^{2} \\
\leq & \left(\sum_{s=1}^{S}\left|i^{N} b_{N}(k a) Y_{N}^{N *}\left(\theta_{s}, \varphi_{s}\right)\right| \times\left|W\left(\theta_{s}, \varphi_{s}\right)\right|\right)^{2} \\
\leq & \sum_{s=1}^{S}\left|i^{N} b_{N}(k a) Y_{N}^{N *}\left(\theta_{s}, \varphi_{s}\right)\right|^{2} \times \sum_{s=1}^{S}\left|W\left(\theta_{s}, \varphi_{s}\right)\right|^{2} \\
= & \left|b_{N}(k a)\right|^{2} \sum_{s=1}^{S}\left|Y_{N}^{N *}\left(\theta_{s}, \varphi_{s}\right)\right|^{2} \times \sum_{s=1}^{S}\left|W\left(\theta_{s}, \varphi_{s}\right)\right|^{2}
\end{aligned}
$$

So:

$$
\sum_{s=1}^{S}\left|W\left(\theta_{s}, \varphi_{s}\right)\right|^{2} \geq \frac{\left|Y_{N}^{N *}\left(\theta_{0}, \varphi_{0}\right)\right|^{2}}{\left|b_{N}(k a)\right|^{2} \sum_{s=1}^{S}\left|Y_{N}^{N^{*}}\left(\theta_{s}, \varphi_{s}\right)\right|^{2}}
$$

Therefore, to have an optimal solution of $\mathbf{W}$, we just set a constraint:

$$
b_{n}(k a) \geq-30 \mathrm{~dB} \text {. }
$$

The linear system (12) then becomes:

$$
\mathbf{A} \times \mathbf{W}=\tilde{\mathbf{B}},
$$
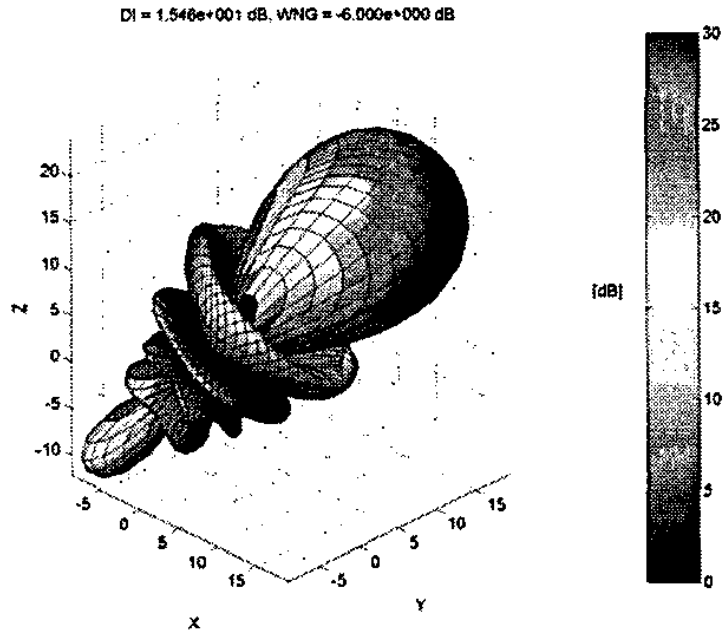

Fig. 4. The optimized beampattem of order five at $2 \mathrm{kHz}$. (DI $=$ $15.46 \mathrm{~dB}, W N G=-6 \mathrm{~dB}$ )

where $\tilde{\mathbf{B}}$ is $\mathbf{B}$ modulated by a step function $\mathcal{U}_{n}(k a)$ :

$$
\begin{aligned}
& \tilde{\mathbf{B}}=\left[\begin{array}{c}
Y_{0}^{0 *}\left(\theta_{0}, \varphi_{0}\right) \times \mathcal{U}_{0}(k a) \\
Y_{1}^{-1 *}\left(\theta_{0}, \varphi_{0}\right) \times \mathcal{U}_{1}(k a) \\
\cdots \\
Y_{N}^{N *}\left(\theta_{0}, \varphi_{0}\right) \times \mathcal{U}_{N}(k a) \\
\ldots \\
Y_{\infty}^{\infty *}\left(\theta_{0}, \varphi_{0}\right) \times \mathcal{U}_{\infty}(k a)
\end{array}\right], \\
& \mathcal{U}_{n}(k a)=\left\{\begin{array}{ll}
1 & b_{n}(k a) \geq-30 \mathrm{~dB} \\
0 & \text { otherwise }
\end{array} .\right.
\end{aligned}
$$

Because of the convergence of the sound field expansion (5), the modulated linear system (22) actually contains finite equations.

The optimization can be numerically solved with MATLAB function fmincon.

\section{RESULTS}

We demonstrate our system by simulations and experiments.

We first plot the beampatterns of order five at $2 \mathrm{kHz}$. We steer the beampattern in $\left(\theta_{0}, \varphi_{0}\right)=(\pi / 4, \pi / 4)$ direction. Fig. 3 shows the distorted beampattern without optimization. The WNG is about $-12.73 \mathrm{~dB}$ which damages the robustness. The optimal beampattern is shown in Fig. 4. It optimally approximates the standard beampattern of order five in the least square sense. This minimizes the irregularities inherent with the system. And it is under the WNG constraint of $-6 \mathrm{~dB}$. As an example, Fig. 5 shows the directivity index (DI) and WNG of an optimal spherical beamformer pointing to the same direction in a wide frequency range. Here the DI is defined as [12]:

$$
D I=10 \log _{10}\left(\frac{\left|H\left(\theta_{0}, \varphi_{0}\right)\right|^{2}}{\frac{1}{4 \pi} \int_{0}^{\pi} \int_{0}^{2 \pi}|H(\theta, \varphi)|^{2} \sin (\theta) d \varphi d \theta}\right),
$$




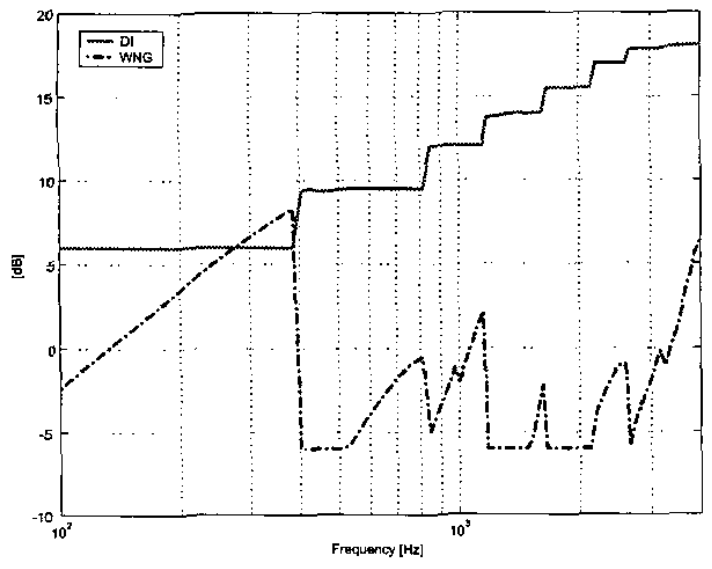

Fig. 5. Optimal spherical beamformer with minimum WNG of $-6 \mathrm{~dB}$.

here $H(\theta, \varphi)$ is the optimized beampattern. The optimal spherical beamformer for all angles in $3 \mathrm{D}$ space can be precomputed and stored in a lookup table.

We then took the spherical microphone array system to roadside to record real-world traffic auditory scenes. A simple scenario is as shown in Fig. 6 where a car is moving from the left side of the array to the right side along the street. To track the car in this scenario from the recordings, for each frame, we simply steer the spherical beamformer to every $3 \mathrm{D}$ directions to search the peaks. We assume the sound from the car is stronger than the sound from all other directions. So the peaks indicate the car locations. Since the spectrum of the sound from the car is not constant, the localization is performed at multiple frequencies. We will extend this to multiple sound source localization later in this section. The tracking path is shown in Fig. 7 where the left and right sides of the array comespond to azimuth angle $\pi$ and 0 , respectively. The jitters on the tracking path may be caused by several factors:

1) the sound spectrum from the vehicle is not constant across the recording time;

2) the sound source is not simply a point source since many parts of a running vehicle can make sound;

3) the environment itself is very noisy with wind blowing, people walking and talking, a building being constructed on the other side of the street, the reflections from walls and the ground surface in addition to self noise of the system.

When the car is far away from the array, its sound fades into the environmental noise. So the jitters tend to be more salient at the two endpoints of the path. We will see this more clearly in the two-vehicle case later.

To show the actual $3 \mathrm{D}$ localization performance of the spherical beamformer, we pick three typical frames of the recorded sound as numbered 1-3 in Fig. 6. The localization result is a $3 \mathrm{D}$ surface, where each surface point represents a localization angle and its distance from the origin represents

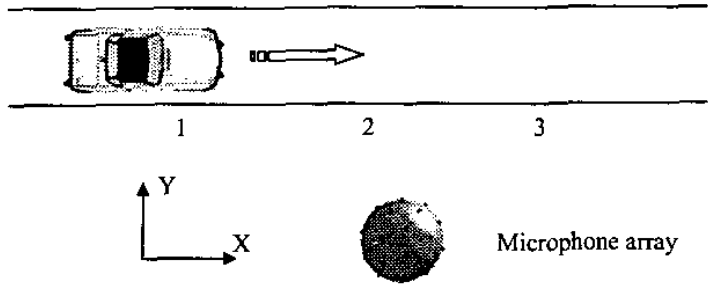

Fig. 6. The scenario of the experiment. A car is moving from left to right. The spherical microphone array is placed on the roadside.

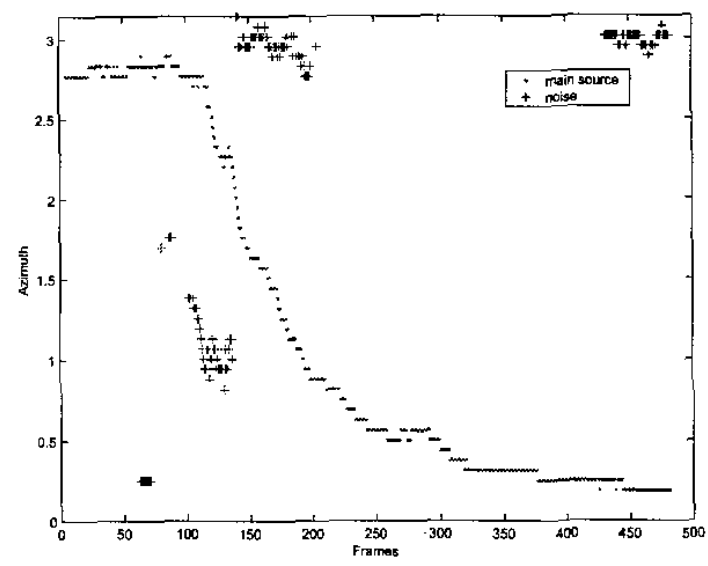

Fig. 7. Tracking one vehicle using the spherical microphone array.

the amplitude of the beamformed signal. In an ideal free space environment under plane wave assumption, using a standard beampattern, the localization surface will have the same shape of this beampattern pointing to the plane wave direction. In a real-world environment, however, those factors listed above will affect the final result which may in turn provide some spatial cues about the sound source and the environment. For clarity, we only show the localizations using a single frequency at about $2 \mathrm{kHz}$, and plot in normalized linear scale instead of $\mathrm{dB}$ scale. We provide two different viewpoints for each figure, the left plot is from the same viewpoint as in Fig. 6 while the right one is from an appropriate $3 \mathrm{D}$ viewpoint. The first $3 \mathrm{D}$ localization is shown in Fig. 8. It has a spindle-like shape. The localization for the second chosen frame is shown in Fig. 9, which indicates the effect of a spreading sound source at this frequency. In addition, the irregular sidelobes are likely caused by all kinds of noise. Fig. 10 shows the 3D localization for the third chosen frame. It clearly shows a second sound source at this frequency, real or image, but weak. We can also see this sound is from the lower part of the car, possibly from the running wheels or brakes.

We also tested our system in a more challenging scenario. In another experiment, we recorded two cars moving from right to left successively in different speed. Fig. 11 shows the tracks of two cars. 

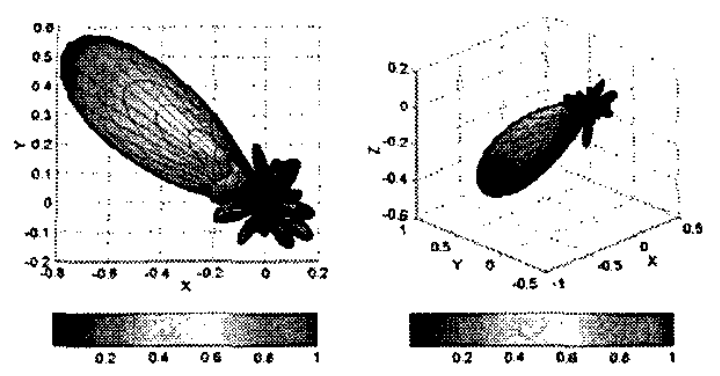

Fig. 8. 3D localization of the first chosen frame. The car is now on the left side of the array.
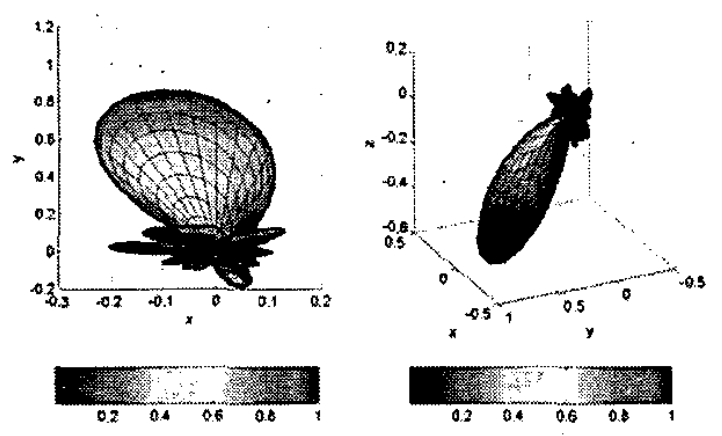

Fig. 9. The car is now roughly in front of the array. The 3D localization has a clam-like shape which indicates the car is approximately a horizontally spreading sound source.

\section{V1. SUMMARY AND FUTURE WORK}

We have built a practical spherical microphone array system using 60 microphones. Our system achieves optimal approximation of the standard beampattern and robustness with the minimum white noise gain of $-6 \mathrm{~dB}$. Just like camera in vision-based traffic surveillance, our spherical microphone array is an analogous tool in sound-based traffic surveillance. We evaluated our system in a real-world traffic environment and demonstrated its performance.

Future work includes visual-audio joint traffic surveillance, complex traffic auditory scene analysis, etc.

\section{REFERENCES}

[1] Y.K. Jung, K.W. Lee and Y.S. Ho "Content-based event retrieval using semantic scene interpretation for automated traffic surveillance", IEEE Transactions on Intelligent Transportation Systems, Vol. 2, No. 3 , pp. $151-163$, Sept. 2001.

[2] H. Veeraraghavan, O. Masoud and N.P. Papanikolopoulos, "Computer vision algorithms for intersection monitoring", IEEE Transactions on Intelligent Transportation Systems, Vol. 4, No. 2, pp. 78-89, June 2003.

[3] T.N. Schoepflin and D.J. Dailey, "Dynamic camera calibration of roadside traffic management cameras for vehicle speed estimation", IEEE Transactions on Intelligent Transportation Systems, Vol. 4, No. 2, pp. 90-98, June 2003.

[4] Y. Chen, "Highway overhead structure detection using video image sequences", IEEE Transactions on Intelligent Transportation Systems, Vol. 4, No. 2, pp. 67-77, June 2003.
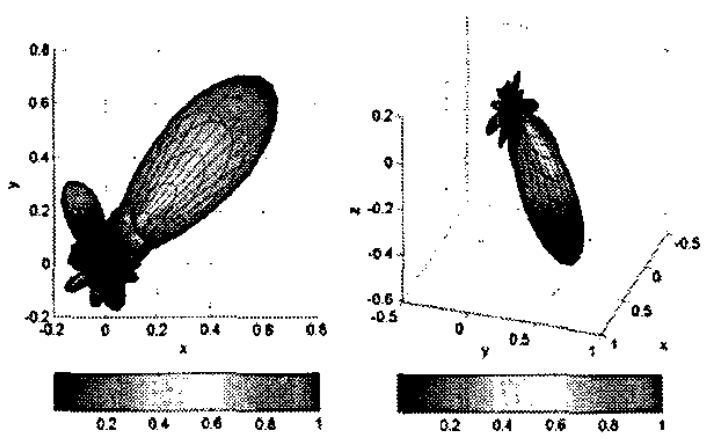

Fig. 10. The car now moves to the right side of the array. The 3D localization has a spindle-like shape again.

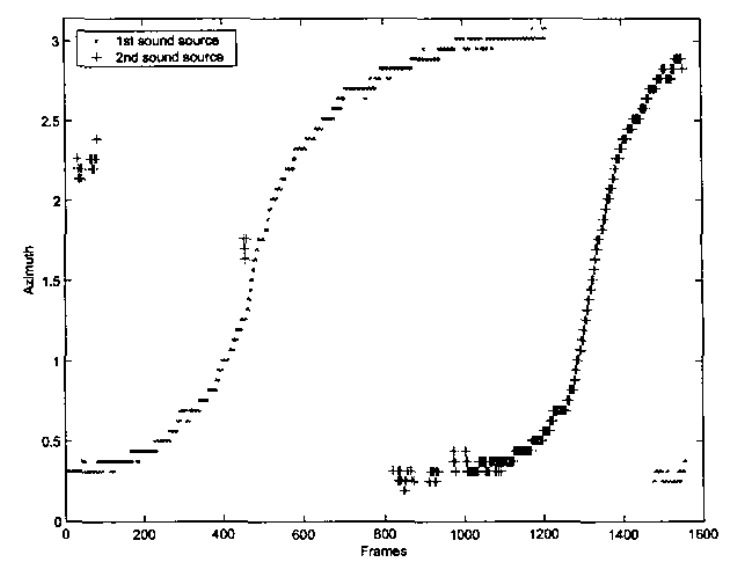

Fig. 11. Tracking two vehicles using the spherical microphone array.

[5] H. Wu, M. Siegel and P. Khosla, "Vehicle sound signature recognition by frequency vector principal component analysis", IEEE Trans. on Instrumentation and Measurement, Vol. 48, No. 5, pp. 1005-1009, October 1999.

[6] http://www.smarteksys.com

[7] Earl G. Williams, Fourier Acoustics, Academic Press, San Diego, 1999

[8] Jens Meyer and Gary Elko, "A highly scalable spherical microphone array based on an orthonormal decomposition of the soundfield", IEEE Proc. ICASSP'02, vol.2, pp. 1781-1784, May, 2002

[9] T. D. Abhayapala and D. B. Ward, "Theory and design of high order sound field microphones using spherical microphone array", IEEE Proc. ICASSP'02, vol.2, pp. 1949-1952, May, 2002

[10] Jörg Fliege and Ulrike Maier: "A Two-Stage Approach for Computing Cubature Formulae for the Sphere". Ergebnisberichte Angewandte Mathematik, No. 139T. Fachbereich Mathematik, Universität Dortmund, 44221 Dortmund, Germany. September 1996.

[11] P.K. Morse, K.U. Ingaard. Theoretical Acoustics, McGraw Hill, New York, 1968.

[12] M. Brandstein and D. Ward (Eds.). Microphone Arrays. SpringerVerlag, New York, 2001.

[13] Zhiyun Li, Ramani Duraiswami, Elena Grassi and Larry S. Davis, "Flexible layout and optimal cancellation of the orthonomality error for spherical microphone arrays", IEEE Proc. ICASSP'04, pp. IV 41-44, May, 2004. 